\title{
Comparative Study of Bisection, Newton, Horner's Method for Solving Nonlinear Equation
}

\author{
Amiruddin Ab Aziz ${ }^{a, *}$, Nur Afriza Baki a, Abdul Zaki Bin Zamri Azuha a, Mohamad Aliff Haziq ${ }^{\text {a }}$ \\ a) Universiti Teknologi MARA (UiTM) - Faculty of Computer and Mathematical Sciences, Terengganu Campus, Malaysia \\ *Corresponding author: amiru2830@uitm.edu.my
}

\section{Paper History}

Received: 01-May-2021

Received in revised form: 01-July-2021

Accepted: 30-July-2021

\begin{abstract}
The Bisection, Newton and Horner's method are used to compare the efficiency to solve nonlinear function such as trigonometric, exponential, logarithmic and cubic polynomial function. Although these methods are considered as alternative, the methods also possess error compared to the exact value. The efficiency is measured by the error produced at the fixed iteration. The methods are converted into $\mathrm{C}$ language and executed by using maple 18 .
\end{abstract}

KEY WORDS: Bisection method, Newton method, Horner's method.

\subsection{INTRODUCTION}

In this study, numerical analysis is the area of mathematics and computer science that creates, analysed and implements algorithms for solving numerically the problems of continuous mathematics [1]. Numerical methods not only provide alternatives in finding solutions to more difficult and complicated mathematical models, but also create algorithms in finding solutions to models that cannot be solved explicitly by the analytical mathematical formula in the most efficient manner.

Based on Artashkin [2], bisection method or sometimes called the method of halving of intervals (also called the Bolzano's method). Relevant situation in Physics where such problems are needed to be solved include finding the equilibrium position of an object, potential surface of a field and quantized energy level of confined structure [3]. The common root-finding methods include Bisection, NewtonRaphson, False position, Secant methods etc.
Different methods converge to the root at different rates. That is, some methods are faster in converging to the root than others. The rate of convergence could be linear, quadratic or otherwise. The higher the order, the faster the method converges [4].

The method is based on the Intermediate Value theorem and attempts to locate a solution to the equation $f(x)=0$ in a sequence of intervals of reducing size [5]. In the Bisection method of solving $f(x)=0$, the function $f(x)$ is assumed to be continuous on the interval $(a, b)$ such that $f(a) * f(b)<0$. Hence, there exists at least one value $r$ in the interval $(a, b)$ in which $f(r)=0$ in which ( $\mathrm{r}$ is the root) [6]. The interval is then halved or divided into two subintervals by the midpoint of $a$ and $b$. Checking is done to locate in which of the two smaller subintervals the root lie. The process is repeated until a desired accuracy is achieved.

Newton's method is also called the Newton-Raphson iteration (attributed to Sir Isaac Newton (1643-1727) and Joseph Raphson (1648-1715) [3]. Being one of the most widely used method of root finding, the procedure attempt to find a solution of the equation $f(x)=0$ where $f(x)$ is a function of one variable continuous and differentiable. In Newton's method, approximation is done by using tangential lines [7]. The solution process begins with choosing a value as the first estimate of the solution (normally obtained from graphing). This initial value is sometimes called the "initial guess". The second estimated is obtained by taking the tangent line to $f(x)$ at the second estimate. The process goes on and on until desired accuracy is achieved.

Horner's method refers to a polynomial evaluation method name after William George Horner [8]. Horner's method used until computers came into general use in about 1970. It was used refer to a root-finding method for polynomials named after Horner who described a similar method in 1819. This method was widely used and became a standard method for hand calculation. It gave a convenient way for using the Newton method for polynomials. It relied on the algorithm for polynomial evaluation now named after Horner [9]. After the introduction of computers this root-finding method went out of use and as a result the term Horner's method has become understood to mean just the polynomial evaluation algorithm. 


\subsection{RESEARCH METHODOLOGY}

There are seven (6) steps involved in this research:

Step 1: First of all, the project is defined for a clear understanding about the main purpose of the project. The root finding method that used to solve nonlinear function are discussed in this step. The method Bisection method, Newton method and Horner's method is chosen for this project. The efficiency of this method is analyzed and compared to Newton method and Bisection method which are among of the best method to solve nonlinear equation. Each method has its own advantages and disadvantages. The efficiency of these method measured by the error produced in the selected iteration.

Step 2: The second step is about studying previous research paper, journals or books that related to this study. The information that benefits this study regarding the bisection, Newton and Horner's method are collected and in order to test out those methods, the nonlinear function must be stated. The four type of function, Trigonometric, Logarithmic, Exponential and Cubic Polynomial are chosen based on the frequent presence in human use or data form.

Step 3: Step discussed the process of define formula of selected methods. Bisection method, Newton's method and Horner's method are studied for further understanding. Thus, the types of function are chosen to be used to those methods.

Step 4: Maple 18 software is used as the place to execute codes. The software is chosen because of simple and easy to apply compare to the other software such C++, MATLAB or BlueJ. The programming codes are implemented with four different functions. An equation is selected for each type of function, Trigonometric, Logarithmic, Exponential and Cubic Polynomial function. The analysis is limited to small interval. The result is compared based on the error produced during the same iteration for each method.

Step 5: The test run of the programming code are executed in order to get the result. If there is an error in the source code, Step 4 is repeated until there is no error detected. The results are collected and recorded in Microsoft Excel. The results are priories the approximation of solution, error at the same number of iteration. The error gains from comparing with the actual solution.

Step 6: The data collected from the test run are tabulated and analyzed. The error of the roots then calculated and stated in the table to make it easier to analyze. The error then compared to get the least error. The method with the least error will be taken as the best method in term of accuracy.

\subsection{IMPLEMENTATION}

\subsection{Implementation of Bisection Method}

Test function:

$$
f(x)=\sin (x)-0.5
$$

\section{Step 1: Identify the interval}

The root of $\mathrm{x}$ for test Function 1 identified as 0.5235987756 . The interval $[0,1]$ is chosen as 0.5235987756 is in between of the number 0 and 1 . The interval is also small interval, which is used to solve the function.

$$
\begin{aligned}
& \text { First point: } f(0) \\
& \begin{aligned}
f(0) & =\sin (0)-0.5 \\
& =-0.5 \text { (Negative) }
\end{aligned}
\end{aligned}
$$

Second point: $f(1)$

$$
\begin{aligned}
f(1) & =\sin (1)-0.5 \\
& =0.3414709848 \text { (Positive })
\end{aligned}
$$

By applying root existence theory:

$$
f(\text { First point }) \times f(\text { Second point })=\text { Negative value }
$$

The points of interval are valid for further calculation.

\section{Step 2: Bisection of interval.}

In bisection method, the initial interval is divided into half and two new interval produced. The calculation for the midpoint is shown below.

By using formula:

$$
\begin{aligned}
& m=\frac{a+b}{2} \\
& m=\frac{0+1}{2} \\
& m=0.5
\end{aligned}
$$

The midpoint is used in the test function. Midpoint $=0.5$ :

$$
\begin{aligned}
f(0.5) & =\sin (0.5)-0.5 \\
& =-0.0205744614
\end{aligned}
$$

There are two intervals now which are $[0,0.5]$ and $[0.5,1]$.

\section{Step 3: Determination of interval with the root existence}

By using the existence of root theory, these two intervals are checked and the interval with the value which is greater than 0 will be chosen as the new interval. The calculation is shown below.

Root Existence Theory:

$$
f(\text { First point }) \times f(\text { Second point })=\text { Negative value }
$$


First interval: $[0,0.5]$

$$
f(0) \times f(0.5)=0.01028723070
$$

Second interval: $[0.5,1]$

$$
f(0.5) \cdot f(1)=-0.007025581596
$$

Since the interval $[0.5,1]$ is obey the root existence theory which is greater than 0 , the interval is chosen to be the new interval for further calculation.

\section{Step 4: Repeat step}

Step 1 to 3 is repeated until the desire number of iteration is reached. When the desire iteration is reached, the error will be evaluated. The evaluation of the error will be shown in 3.4 Error calculation and analysis.

\subsection{Implementation of Newton method}

Newton algorithm is quite different compared to Bisection. Newton method only needs one initial point as the initial guess for next evaluation. Choosing initial point is crucial because if the initial guess is far from the root, it may diverge and the root cannot be found. The implementation is as shown below:

\section{Step 1: Choosing initial point}

The test limited to small interval or close initial point. Since the root for this test function 0.5235987756 , the initial point chosen is 1 .

\section{Step 2: Derivation of test function and point substitution}

Since the derivative of function is needed in the calculation, the derivation of test function computed by using Maple 18 software. The derivative for $f(x)$ is denoted as $f^{\prime}(x)$ and the point substitution is as shown below:

$$
\begin{aligned}
& f(x)=\sin (x)-0.5 \\
& f^{\prime}(x)=\cos (x)
\end{aligned}
$$

Point substitution: 1

$$
\begin{aligned}
& f(1)=\sin (1)-0.5=0.3414709848 \\
& f^{\prime}(1)=\cos (1)=0.5403023059
\end{aligned}
$$

\section{Step 3: Calculation of new root}

Step 3 is the calculation of the new root by using the formula (17).

$$
x_{n}=x_{n-1}-\frac{f\left(x_{n-1}\right)}{f^{\prime}\left(x_{n-1}\right)}
$$

Test function is divided by its derivative. After that, this quotient is subtracted by the old point in order to get the new root. For test function 1, calculation is shown below:

$$
\begin{aligned}
& x_{1}=x_{0}-\frac{f\left(x_{0}\right)}{f^{\prime}\left(x_{0}\right)} \\
& x_{n}=1-\frac{0.3414709848}{0.5403023059} \\
& x_{n}=0.3680001342
\end{aligned}
$$

Then, the first iteration for Newton method is 0.3680001342 .

\section{Step 4: Repeat the step}

Step 1 to step 3 is repeated until a specific number of accuracies is archived. As for the number toward constant in tolerance 0.01 or $10^{-2}$. The calculation continued for the next iteration.

Second iteration:

$$
\begin{aligned}
& x_{2}=0.3680001342-\frac{-0.1402498195}{0.9330486631} \\
& x_{2}=0.5183136441
\end{aligned}
$$

Third iteration:

$$
\begin{aligned}
& x_{3}=0.5183136441-\frac{-0.1402498195}{0.9330486631} \\
& x_{3}=0.5235907857
\end{aligned}
$$

Forth iteration:

$$
\begin{aligned}
& x_{4}=0.5235907857-\frac{-0.0045840200}{0.8686558621} \\
& x_{4}=0.5288679273
\end{aligned}
$$

By forth iteration, the solution has met the constant on tolerance 0.01 .

\subsection{Implementation of Horner's method}

In order to find the approximate real roots for a polynomial, Horner's method need to combine with Newton method. The implementation is as shown below: 
Test Function 1:

$$
p_{6}(x)=x^{6}+4 x^{5}-72 x^{4}+214 x^{3}+1127 x^{2}+1620 x-5040
$$

This can be factor:

$$
(x-3)(x+3)(x+5)(x+8)(x-2)(x-7)
$$

\section{Step 1: Use Newton method to find largest zero}

From above, the largest root of this polynomial is 7 and makes an initial guess of 8 .

Step 2: Using Horner's method $p(x)$ is divided by $(x-7)$ to obtain

$$
p_{5}(x)=x^{5}+11 x^{4}+5 x^{3}-179 x^{2}-126 x+720
$$

\section{Step 3: Repeat the step}

Step 1 and step 2 is repeated until all the expected roots were found.

\subsection{Error calculation and analysis}

There exist a few contrasts in the middle of the math directed by adding machine or PC and the number juggling in analytics and variable based math courses [10]. These various qualities are called error where it is not quite the same as the accurate esteem. The approximated roots are arranged and contrast with the genuine roots. Blunder computation is determined by utilizing the equation underneath by utilizing Maple 18 and Microsoft Excel 2016. The littlest rate error is considered as the most exact to the genuine esteem and demonstrated that the strategy and the resilience are the most dependable.

Error $=\left|\frac{(\text { actualvalue }- \text { approximationvalue })}{\text { actualvalue }}\right|$

\subsection{RESULT AND DISCUSSION}

\subsection{Numerical results}

Table 1: Error produced by the Three Methods on the Test Function.

\begin{tabular}{|c|c|c|c|}
\hline & \multicolumn{3}{|c|}{ Method } \\
\hline $\begin{array}{c}\text { Test } \\
\text { Function }\end{array}$ & Bisection & Newton & Horner \\
\hline 1 & $7.97 \times 10^{-3}$ & $1.0 \times 10^{-10}$ & Failed \\
\hline 2 & $2.5 \times 10^{-2}$ & $1.0 \times 10^{-9}$ & Failed \\
\hline 3 & $3.1 \times 10^{-2}$ & 0 & 0 \\
\hline 4 & $2.9 \times 10^{-2}$ & $4.0 \times 10^{-10}$ & Failed \\
\hline
\end{tabular}

Essentially, every one of the three methods is utilized to approximate root of a capacity for nonlinear functions. For this situation, exponent, logarithmic, trigonometric and cubic polynomial functions were picked to test out every one of the three methods. This outcome is on the count kept running by Maple 18. At that point, these methods thought about dependent on the error delivered from the iteration picked.

From the Table 1, the least error produced for all the test function is the Newton's method. Thus, the best method in term of accuracy is the Newton's method because it is produced least error compared to the Bisection and Horner's methods.

\subsection{CONCLUSION}

From the numerical result, the stated error was stated in the Table 1 which lead to the conclusion of the most reliable and accurate method to be used. With the least amount of error produced during the fifth iteration, Newton's method is proven as the most reliable method among the three methods in term of accuracy.

\section{REFERENCES}

[1] Boyd, S., Balakrishnan, V., \& Kabamba, P. (1989). A bisection method for computing the $\mathrm{H}$ norm of a transfer matrix and related problems. Mathematics of Control, Signals and Systems, 2(3), 207-219.

[2] Artashkin, E.P. (2016). BISECTION METHOD. APRIORI. CЕРИЯ: ЕСТЕСТВЕННЫЕ И ТЕХНИЧЕСКИЕ НАУКИ, (6), 4.

[3] Iwetan, C.N., Fuwape, I. A., Olajide, M.S., \& Adenodi, R.A. (2012). Comparative Study of the Bisection and Newton Methods in solving for Zero and Extremes of a Single-Variable Function. J. of NAMP, 21, 173-176.

[4] Charles, J. (2008). Comparative Study of Bisection, Newton-Raphson and Secant. Comparative Study of Bisection, Newton-Raphson and Secant - Root Finding Method.

[5] Matveev, M.N. (2016). An intermediate value theorem for face polytopes. Lobachevskii Journal of Mathematics, 37(3), 307-315. https://doi.org/10.1134/S1995080216030173.

[6] Namekar, S. (2019). Role of Bisection Method. International Journal for Research in Applied Science and Engineering Technology, 7(11), 426-429. https://doi.org/10.22214/ijraset.2019.11068.

[7] Zaslavski, A. J. (2016). Newton's method. In Springer Optimization and Its Applications (Vol. 108, pp. 265296). https://doi.org/10.1007/978-3-319-30921-7_16.

[8] He, T.-X., \& Shiue, P. J.-S. (2012). A note on Horner's method. J. Concr. Appl. Math., 10(1-2), 53-64.

[9] Muller, J.-M., \& Muller, J.-M. (2016). Polynomial Evaluation. In Elementary Functions (pp. 81-100). https://doi.org/10.1007/978-1-4899-7983-4_5.

[10] Burden, A., L. Burden, R., \& Douglas Faires, J. (2016). Numerical Analysis, 10th ed. Communications of the ACM (Vol. 10). https://doi.org/10.13140/2.1.4830.2406. 